\title{
Linking pathways in colorectal cancer
}

\author{
Gijs R van den Brink
}

Studies of inherited cancer syndromes have implicated numerous signaling pathways in colorectal carcinogenesis, but the relationship between these signaling pathways remains poorly understood. Now, a new mouse model of juvenile polyposis syndrome identifies a molecular mechanism for clonal epithelial cell expansion and links several pathways with an established role in polyposis.

In recent years, we have learned much about the molecular pathways involved in intestinal polyp formation and colorectal carcinogenesis by studying families with rare autosomal dominant cancer syndromes. But despite progress in identifying the relevant pathways, our understanding of how these pathways regulate intestinal stem cell (ISC) behavior remains limited. On page 1117 of this issue, $\mathrm{Xi} \mathrm{He}$ and colleagues ${ }^{1}$ make some important observations using a new mouse model of juvenile polyposis syndrome (JPS), an inherited cancer syndrome resulting from loss-of-function mutations in BMP pathway components $^{2,3}$. Their data provide insights into the way ISC numbers are controlled and increase our understanding of the mechanism of crypt fission, an important phenomenon underlying the clonal expansion of intestinal tumors.

\section{Pathways to polyposis}

Intestinal polyps arise from single epithelial precursor cells by clonal expansion of their progeny ${ }^{4}$. An increase in the population of affected daughter cells occurs through multiplication (fission) of affected intestinal crypts and spreading of cells over the superficial epithelium, with overgrowth into unaffected neighboring crypts (Fig. 1a,b). Classically, intestinal polyps are categorized into two classes based on histology. Adenomatous polyps are neoplasms showing clonal expansion of dedifferentiated epithelial cells and are considered precursors to adenocarcinomas. Hamartomatous polyps, in contrast, are lesions in which expansion of epithelial cells coincides with expansion of other mucosal cell types. Unlike adenomatous polyps, epithelial cells in hamartomatous polyps seem to differentiate normally and are not necessarily neoplastic in nature, although secondary adenomatous transformation does occur in these polyps.

Gijs R. van den Brink is in the department of Experimental Internal Medicine of the Academic Medical Center, Amsterdam, the Netherlands. e-mail:g.r.vandenbrink@amc.uva.nl
JPS is a rare autosomal dominant syndrome characterized by development of multiple hamartomatous polyps throughout the gastrointestinal tract. Some families with JPS carry mutations in the genes encoding the $\mathrm{BMP}$ receptor BMPR1A and its signaling intermediate SMAD4 (refs. 2,3). As BMP signaling has been shown to regulate apoptosis of intestinal epithelial cells $s^{5}$, mutations in BMP pathway components could interfere with programmed cell death, resulting in the epithelial overgrowth observed in JPS. But the mechanism underlying the increase in the rate of crypt fission in JPS remains unknown. The new work by $\mathrm{He}$ et al. ${ }^{1}$, together with a recent study by Haramis et al. ${ }^{6}$, sheds light on this issue and identifies some of the molecular pathways controlling crypt fission. Their findings not only are relevant for our understanding of JPS but also provide insights into the cellular behavior of sporadic (noninherited) polyps, precursors to the second most prevalent cause of cancer death in the Western world.

\section{Linking pathways}

He et al. used a conditional approach in mice to shut down BMP signaling in the intestinal epithelium. The mice had hamartomatous polyps similar to those seen in individuals with JPS, with amplification of epithelial precursor cell numbers and an increased rate of crypt fission. The authors then focused on the molecular mechanisms underlying this pre-

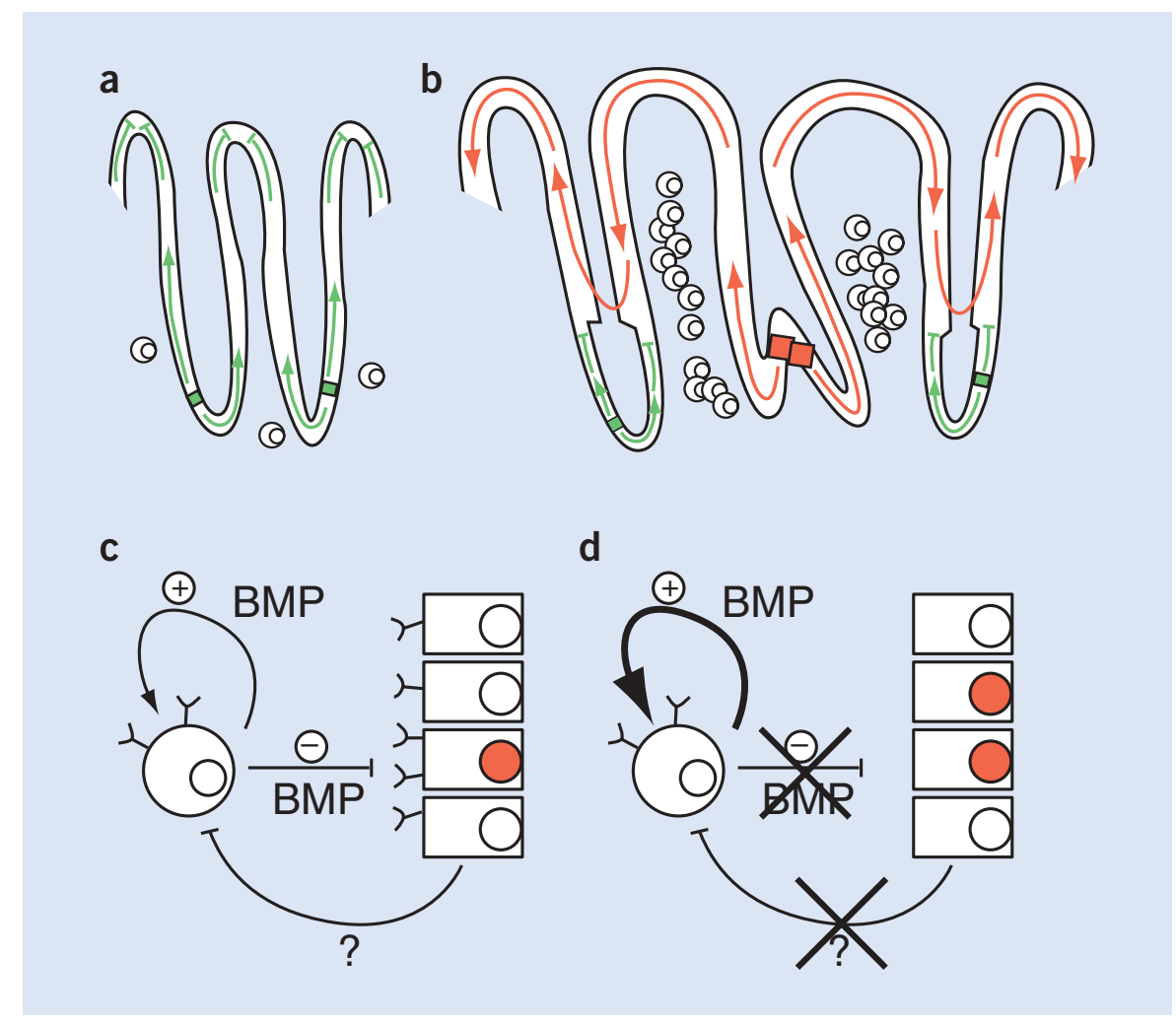

Figure 1 Cellular behavior in JPS polyps. (a) Normal ISCs (green) supply a single crypt. (b) In polyps, mutant ISCs (red) spread clonally through crypt fission and overgrowth of adjacent crypts. (c,d) A model of BMP-Wnt interaction in normal (c) and JPS (d) intestinal crypts. $\beta$-catenin-positive ISC nuclei are shown in red. 
cursor cell expansion and observed that both phosphatidyl inositol 3' (PI3) kinase-AKT and $\beta$-catenin-T-cell factor (TCF) signaling seem to occur exclusively in ISCs. They further showed that, in Bmprla-negative polyps, the number of ISCs is greatly increased. This study presents the first evidence that these three pathways may be directly linked in regulating ISC expansion.

There are two notable aspects to the authors' model. The first is that it links BMP signaling with $\beta$-catenin-TCF signaling. The classical Wnt pathway maintains the epithelial precursor cell phenotype $\mathrm{p}^{7}$ and controls the rate of crypt fission ${ }^{8}$, and deregulated $\beta$ catenin-TCF signaling is the initiating event in most adenomatous polyps ${ }^{9}$. The second notable aspect of the model is that it incorporates PI3 kinase signaling. Germline mutations in PTEN, encoding a negative regulator of the PI3 kinase pathway, have been found in $80-85 \%$ of individuals with Cowden syndrome, a disorder characterized by hamartomatous polyps similar to those seen in JPS ${ }^{10}$.

The authors hypothesize that $\beta$-cateninTCF transcriptional activity in ISCs is activated by the PI3 kinase-Akt pathway, which in turn depends on the inactivation of PTEN by the BMP antagonist Noggin. This model is consistent with a previously described regulatory relationship between
PTEN and BMP signaling ${ }^{11}$. Although activation of $\beta$-catenin through PI3 kinase signaling is somewhat controversial ${ }^{12}$, this activation may be cell-type dependent. Thus, He et al. provide us with a testable model in which three pathways already linked to different polyposis syndromes cooperate to control ISC numbers and the rate of crypt fission.

\section{Tissue interactions}

One of the defining aspects of JPS that has not been addressed by either Haramis et al. or $\mathrm{He}$ et al. is the question of why lamina propria cells show increased proliferation in the hamartomatous polyps of individuals with JPS. The polyps in these individuals can show metaplasia of lamina propria cell types, including the formation of ectopic bone or cartilage. This indicates that BMP signaling in the lamina propria may actually be enhanced, as overexpression of BMP2 in the developing gut has previously been reported to result in ectopic cartilage formation ${ }^{13}$. Lack of BMP signaling in the intestinal epithelium may lead to enhanced production of BMPs in the lamina propria or epithelial cells owing to the resulting loss of a normal feedback inhibitor (Fig. 1c,d). It has been reported that BMP2 levels are increased in the intestines of mice treated with the BMP antagonist Noggin ${ }^{5}$.
High concentrations of BMPs probably stimulate growth of lamina propria cells (Fig. $\mathbf{1 c}, \mathbf{d})$, as BMPs have previously been shown to stimulate proliferation of hematopoietic cells $^{14}$ and stromal cell types in the kidney ${ }^{15}$.

In conclusion, the studies by Haramis et al. and $\mathrm{He}$ et al. suggest that there is an important interplay between BMP and Wnt signaling in the control of ISC number and crypt branching. Some interesting lessons about epithelial-mesenchymal interactions may still be learned from their animal models of JPS.

1. He, X. et al. Nat. Genet. 36, 1117-1121 (2004).

2. Howe, J.R. et al. Science 280, 1086-1088 (1998).

3. Howe, J.R. et al. Nat. Genet. 28, 184-187 (2001).

4. Preston, S.L. et al. Cancer Res. 63, 3819-3825 (2003).

5. Hardwick, J.C. et al. Gastroenterology 126, 111-121 (2004).

6. Haramis, A.P. et al. Science 303, 1684-1686 (2004).

7. van de Wetering, M. et al. Cell 111, 241-250 (2002).

8. Bjerknes, M., Cheng, H., Hay, K. \& Gallinger, S. Am. J. Pathol. 150, 833-839 (1997).

9. Bienz, M. \& Clevers, H. Cell 103, 311-320 (2000).

10. Pilarski, R. \& Eng, C. J. Med. Genet. 41, 323-326 (2004).

11. Waite, K.A. \& Eng, C. Hum. Mol. Genet. 12, 679-684 (2003).

12. Doble, B.W. \& Woodgett, J.R. J. Cell. Sci. 116 1175-1186 (2003)

13. Smith, D.M., Nielsen, C., Tabin, C.J. \& Roberts, D.J. Development 127, 3671-3681 (2000).

14. Detmer, K. \& Walker, A.N. Cytokine 17, 36-42 (2002).

15. Miyazaki, Y., Oshima, K., Fogo, A. \& Ichikawa, I. Kidney Int. 63, 835-844 (2003). 